\title{
Synchronous Rectal Tumours with Different Molecular and Genetic Phenotypes Occurring in a Patient with Lynch Syndrome
}

\author{
Qiheng Gou' \\ Yuxin Xiel \\ Mengni Zhang ${ }^{2}$ \\ Ye Chen ${ }^{3}$ \\ Yali Shen ${ }^{3}$ \\ 'Department of Head and Neck \\ Oncology, Cancer Center, West China \\ Hospital, Sichuan University, Chengdu, \\ Sichuan, People's Republic of China; \\ ${ }^{2}$ Department of Pathology, West China \\ Hospital, West China Medical School, \\ Sichuan University, Chengdu, Sichuan, \\ People's Republic of China; ${ }^{3}$ Department \\ of Abdominal Oncology, Cancer Center, \\ West China Hospital, Sichuan University, \\ Chengdu, Sichuan, People's Republic of \\ China
}

\begin{abstract}
The increasingly widespread use of immunohistochemistry and next-generation sequencing (NGS) in the detection of microsatellite instability (MSI) and DNA mismatch repair (MMR) status has led to the observation of various unusual tumour types that exhibit MMR protein deficiency in Lynch syndrome (LS). Here, we report a case of two synchronous colorectal cancer (CRC) tumours simultaneously occurring in a 42-year-old woman with a deleterious germline mutation in MSH6, abundant expression of PD-L1 and high tumour mutation burden (TMB). The two CRC tumours (tumours A and B) harboured highly heterogeneous features. One showed loss of MSH6 protein and a microsatellite stable (MSS)/ MSI-low (MSI-L) status, while the other presented no loss of MMR protein and MSI-H status. Furthermore, the 9 common mutated genes between the two CRC tumours had no shared mutation sites. Only 4 KEGG pathways were identified as enriched for five of the common mutated genes, while 8 cancer-related pathways were identified as enriched for 9 and 13 unique mutated genes in tumours A and B, respectively. Therefore, we chose immune checkpoint inhibitors (ICIs) as the potential therapy. This case exemplifies the complexity of tumorigenesis and potential ICI treatment in LS patients.
\end{abstract}

Keywords: lynch syndrome, colorectal cancer, heterogeneity, microsatellite instability, immune checkpoint inhibitors

\section{Plain Language Summary}

The increasingly widespread use of immunohistochemistry and next-generation sequencing in the detection of microsatellite instability (MSI) and DNA mismatch repair (MMR) status has led to the observation of various unusual tumour types that exhibit MMR protein deficiency in Lynch syndrome. Here, we report a case of two synchronous colorectal cancer tumours simultaneously occurring in a 42-year-old woman with a deleterious germline mutation in MSH6, abundant expression of PD-L1 and high tumour mutation burden. The two CRC tumours harboured highly heterogeneous features. This case exemplifies the complexity of tumorigenesis and potential immune checkpoint inhibitors treatment in LS patients.

\section{Introduction}

Colorectal cancer (CRC) is the third-most common type of cancer in China. ${ }^{1}$ Lynch syndrome (LS) is the most common autosomal dominant CRC susceptibility syndrome, accounting for $1-3 \%$ of all CRC cases. ${ }^{2}$ Individuals with LS-related CRC exhibit several characteristics that differ from those who have sporadic CRC. 
Tumours harbouring a deficient mismatch repair (MMR) system owing to germline, somatic or epigenetic inactivation account for $20 \%$ of CRC tumours. ${ }^{3}$

LS is caused by pathogenic germline mutations in any one of four key DNA MMR genes: $60-80 \%$ of cases are caused by MLH1 and MSH2 germline mutations, a small proportion of cases are caused by MSH6 and PMS2 germline mutations, and EpCAM germline mutations are rare. ${ }^{4}$

In addition to the continuous advancement of the understanding of the clinical phenotype of LS, the genetic characteristics and tumour mutation burden (TMB) information provided by second-generation sequencing can be used as new biomarkers of LS. In particular, microsatellite instability (MSI) has also been found to be an important biomarker predicting a lack of benefit from fluoropyrimidine monotherapy ${ }^{4,5}$ but a benefit from anti-programmed cell death-1 (PD-1) therapy ${ }^{6,7}$ for CRC.

Only a few mutations are shared amongst most colorectal tumours, and each tumour has a unique combination of genetic alterations. ${ }^{8}$ Therefore, it is likely that no 2 CRC tumours are exactly alike, a daunting consideration for rational planning for treatment.

With the goal of better understanding the occurrence and treatment of CRC in LS patients, we herein report a case of a woman who carried a pathogenic germline mutation in the MSH6 gene and simultaneously developed 2 highly heterogeneous tumours, one of which was MSS/ MSI-L and the other of which was MSI-H. The clinical, pathologic and genetic findings of our case are presented.

\section{Case Presentation}

A 42-year-old woman diagnosed with rectal adenocarcinoma had 2 distinct masses in the rectum: one was $4 \mathrm{~cm}$ from the anus (mass A), and the other was $5-8 \mathrm{~cm}$ from the anus (mass B). A preoperative colonoscopic biopsy was performed, and the diagnosis was adenocarcinoma.

The patient subsequently underwent extended radical resection of the rectal tumours, and the postoperative pathological stage was as follows: AJCC 8th edition pTNM stage pT3N0M0. The histologic examination findings of mass A were consistent with invasive carcinoma (pT3). The tumour was $3.5 \mathrm{~cm}$ in greatest dimension and had features most similar to those of adenocarcinoma. Notably, the tumour did not exhibit vascular invasion, nerve invasion, invasions of the vessels outside the intestinal wall or lymphatic metastasis on immunohistochemistry (IHC) assessment (0/17). The Ki67 IHC result was $90 \%$. Mass $\mathrm{B}$ also showed features of invasive adenocarcinoma on histologic examination, as did mass $\mathrm{A}$, and was $6 \mathrm{~cm}$ in greatest diameter. The two masses were found to be highly heterogeneous through IHC, nextgeneration sequencing (NGS) and tumour immune microenvironment (TIME) analyses.

Notably, the two masses harboured completely different MMR/MSI statuses according to IHC and NGS analyses. Mass A demonstrated deficient MMR (dMMR); specifically, there was an absence of MSH6 protein but normal expression of MLH1, MSH2 and PMS2. Mass $\mathrm{B}$ expressed all $4 \mathrm{MMR}$ proteins and was therefore deemed to have proficient MMR (pMMR) (Figure 1). Furthermore, the mass A was MSS/MSI-L and had high TMB (60.93 mutations/Mb, percentage: $99.33 \%$ ); in contrast, mass B was MSI-H and had high TMB (53.3 mutations $/ \mathrm{Mb}$, percentage: $99.22 \%$ ). Germline mutation analysis of the MMR genes identified the c.1483C $>\mathrm{T} p$. R495* mutation in MSH6 and the KRAS p.G13D mutation in mass $\mathrm{A}$ and the p.G12D mutation in mass B; however, BRAF V600E mutations were not detected in either tumour. Notably, previous studies showed that $93 \%$ of cases have dMMR and are MSI-H. If only the MSH6 protein is absent, the MSI status might be MSI or MSS. However, approximately $7 \%$ of cases show inconsistent characteristics. $^{9-11}$ Thus, we detected that the two masses harboured very different, and even inconsistent, MMR/ MSI statuses.

The two masses were found to have genetic heterogeneity under further sequencing. By NGS, the tumours harboured 3 gene mutations with strong clinical significance in treatment, prognosis or diagnosis, 3 gene mutations with potential clinical significance, and 115 gene mutations with unknown clinical significance. Notably, the 9 mutually mutated genes between the two CRC tumours had no identical mutation sites. This finding highlighted that the two tumours were highly heterogeneous. The genetic features of the two tumours were also highly heterogeneous. Only 4 KEGG pathways, including the RTK/RAS, NOTCH, WNT and TGF beta pathways, were enriched by five of the mutually mutated genes. In contrast, we found that the 2 tumours harboured at least 22 unique mutated genes ( 9 in tumour A vs 13 in tumour B), such as ATM, EGFR, CDKN2A, ALK, ROS1, and NTRK1. These mutations showed enrichment of 8 cancer-related KEGG pathways, including the RTK/RAS, Notch, PI3K, P53, cell cycle, Hippo, Wnt, and TGF beta pathways. Therefore, the patient harboured two highly heterogeneous masses with an inconsistent MMR/MSI status. 

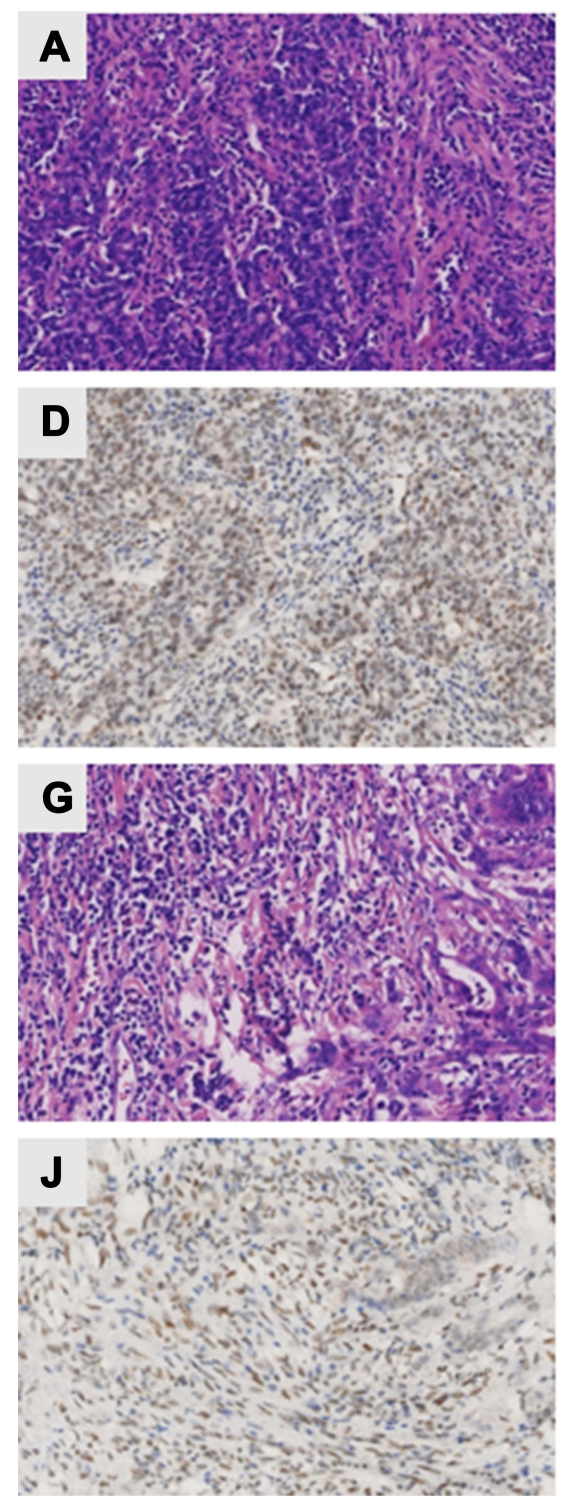
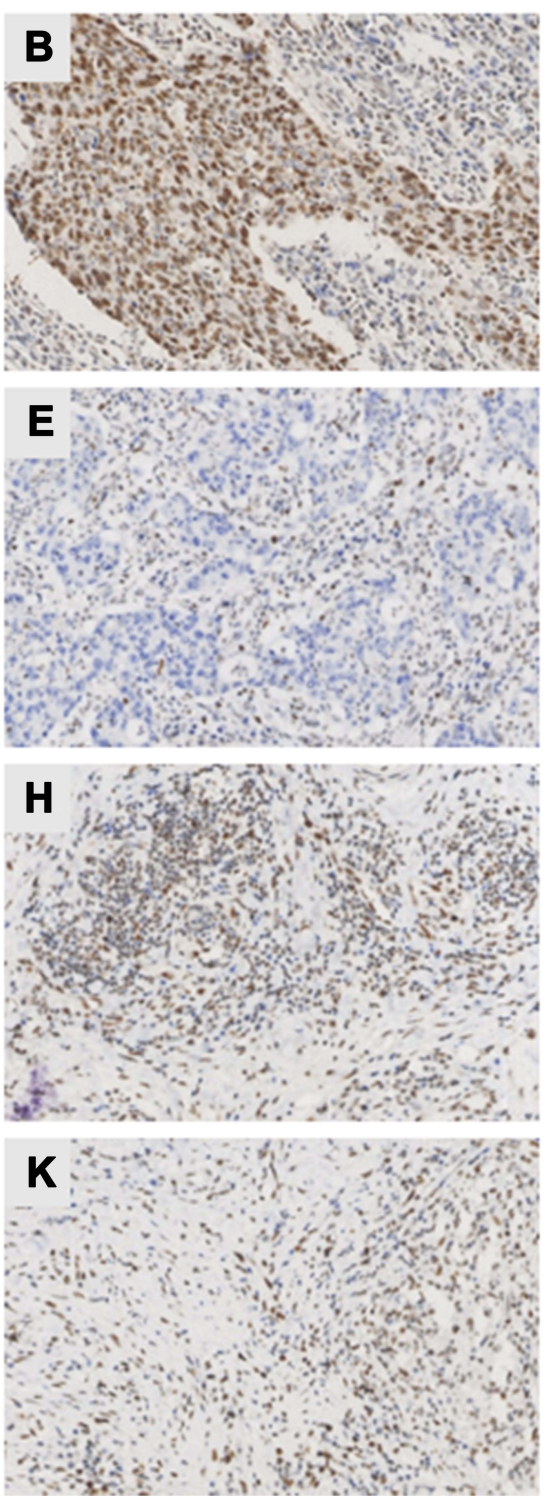
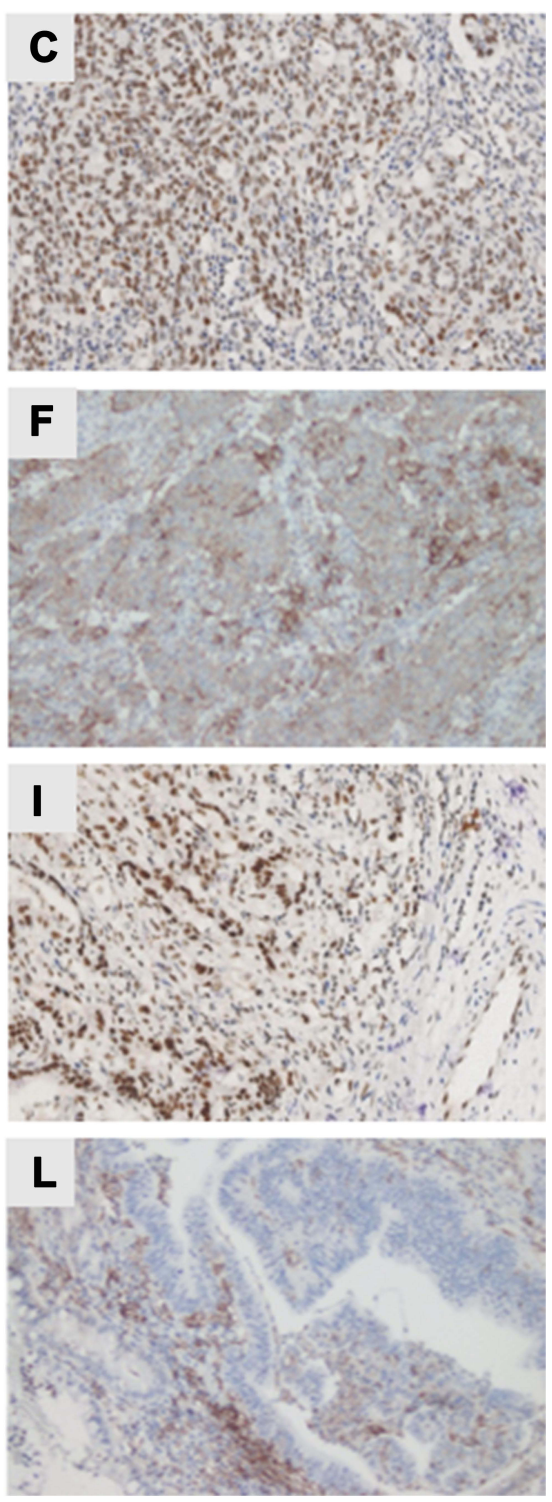

Figure I Heterogeneous morphology, MMR protein expression and PD-LI expression patterns of the 2 synchronous rectal tumours (masses A and B) in the LS patient. (A and G) HE staining of masses A and B showing the classic protruding pattern of adenocarcinoma growth. The IHC staining of mass A showed intact expression of MLHI (B), PMS2 (C), MSH2 (D), and PD-LI (F) in addition to loss of expression of MSH6 (E). Mass \#B showed intact expression of MLHI (H), PMS2 (I), MSH2 (J), MSH6 (K) and PD-LI (L). All images were collected at 100× magnification.

Furthermore, we speculated that the patient was eligible for immune checkpoint inhibitor (ICI) strategies. Both masses had positive programmed cell death ligand 1 (PDL1) (Ventana, clone SP142) expression. Mass A showed predominant positive (3+) PD-L1 expression, including $70 \%$ positivity for tumour cells and $3 \%$ positivity for immune cells. Mass B presented strong positive PD-L1 expression; however, it had less than $1 \%$ positivity for tumour cells and $5 \%$ positivity for immune cells (Figure 1). On the other hand, the combined positive score (CPS) results for masses $\mathrm{A}$ and $\mathrm{B}$ were 30 and 5, respectively. In particular, the CD8 $+\mathrm{T}$ cell proportions in the two CRC tumours were $9.07 \%$ and $6.63 \%$, respectively (Figure 3). In addition to the MSI-H and high TMB statuses, there were $\mathrm{CD} 8^{+} \mathrm{PD}-1^{+} \mathrm{T}$ cells and $\mathrm{CD} 68^{+} \mathrm{PD}-$ $\mathrm{L} 1^{+}$macrophages (Figure 3). The HLA-I molecules were of the hla-b44 supertype, which are positive indicators for immunotherapy. Considering the numerous markers indicating the potential of immunotherapy, including MSI-H, high TMB, presence of $\mathrm{CD} 8^{+} \mathrm{PD}-\mathrm{L} 1^{+}$cells and $\mathrm{CD} 68^{+} \mathrm{PD}-$ $\mathrm{L}^{+}$macrophages, and the HLA-b44 supertype, ICI strategies had the potential to be effective.

The patient's family history fulfilled the Amsterdam II criteria. Specifically, the patient's mother, brother and 


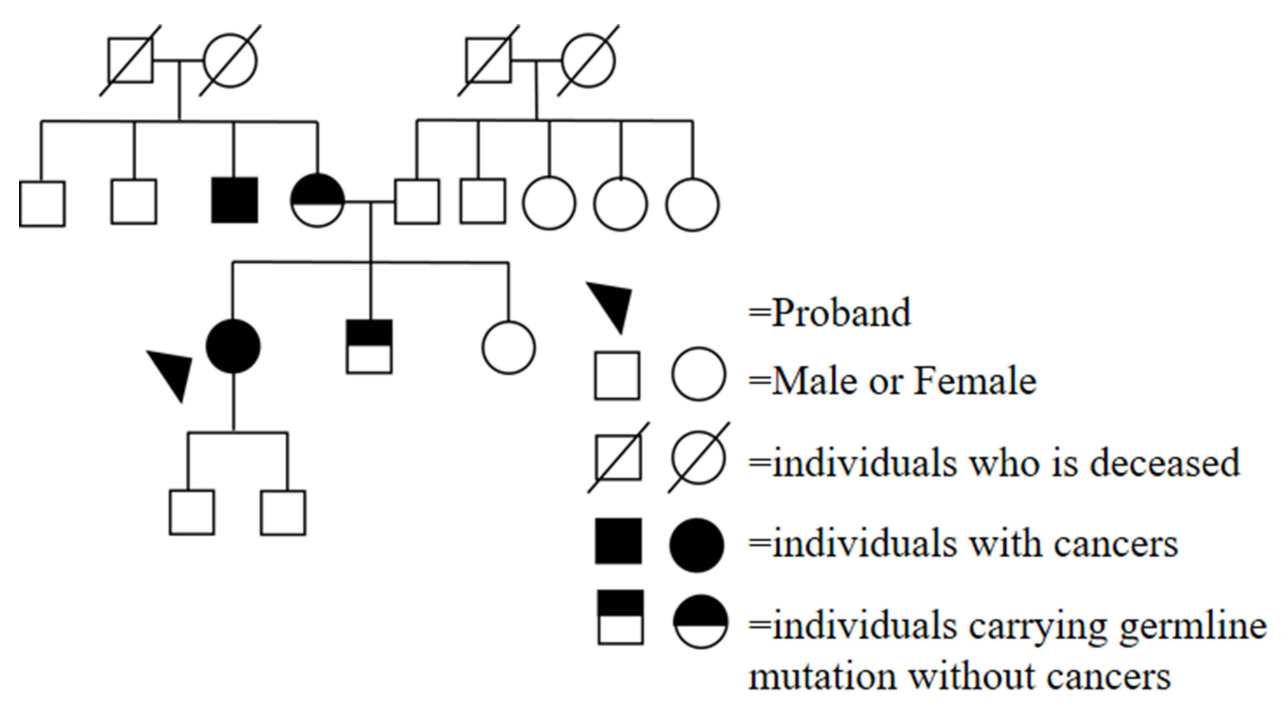

Figure 2 The patient's pedigree. The proband had rectal cancer and carried the MSH6 p.R495* germline mutation. Similarly, the individuals (the patient's mother and the patient's brother) without cancers carried the MSH6 p.R495* germline mutation. One of the patient's uncles had colon cancer but did not undergo NGS assessment.

sister agreed to undergo NGS assessment. Both the patient's mother and brother, but not her sister, were found to have the MSH6 p.R495* germline mutation (similar to the patient). In addition, one of the patient's uncles was affected by colon cancer without detailed information (Figure 2). Given the patient's MMR status and family history, she was diagnosed with LS.

Adjuvant treatments included pelvic radiotherapy and chemotherapy in the form of three cycles of the XELOX regimen. The patient has remained alive up to the writing of this manuscript (April 10, 2021) without tumour recurrence or metastasis. The institutional review board of West China hospital, Sichuan University approved the use of health data in this research, and approved the publication of this case report. And informed consent was also obtained from the patient and the family for the publication of this case report.

\section{Discussion}

LS offers an illustrative example of substantial progress that has been made in understanding the genetic basis of human diseases with technical developments. LS is well defined in terms of specific genes, and the genetic challenges that remain mainly concern optimal detection methods and the prognosis of variant-specific therapies. In our case, the patient with LS had two heterogeneous colorectal tumours. Patient with CRC are recommended to undergo systematic screening for LS, consisting of MSI and MMR assessment, followed by further appropriate analyses, including $B R A F-\mathrm{V} 600 \mathrm{E}$ mutation and germline mutation testing. ${ }^{12}$ This "universal" screening approach, especially considering its use of high-throughput sequencing, which is gradually replacing single protein-based methods, is likely to multiply the total numbers of MMR gene mutations that need to be annotated for pathogenic significance in germline mutation detection. ${ }^{13}$

Previous studies about anti-PD-1 therapy in advanced dMMR CRC have shown a response rate of only approximately $32-53 \%$. $^{6,7,14}$ Primary resistance, which is closely related to the TIME, especially tumour infiltrating lymphocytes (TILs), is one of the main reasons for the failure of ICI treatment. ${ }^{15,16}$ The TIL content can reveal prognostic and immunotherapy response information for cancers. In particular, increased abundances $\mathrm{CD}^{+} / \mathrm{CD}^{4+} \mathrm{T}$ cells, $\mathrm{CD}^{2} 5 \mathrm{RO}^{+} \mathrm{T}$ cells, M1 macrophages, mature dendritic cells (DCs) and natural killer (NK) cells in the tumour area and stroma but a decreased abundance of infiltrating regulator $\mathrm{T}$ (Treg) cells indicates a generally positive prognosis and satisfactory effectiveness of immunotherapy in cancers; the oppose relationships are also true. ${ }^{17}$ Our data showed that $\mathrm{CD} 8^{+} \mathrm{T}$ cells, $\mathrm{CD} 8^{+} \mathrm{PD}-1^{+} \mathrm{T}$ cells and $\mathrm{CD} 8^{+} \mathrm{PD}-\mathrm{L1}^{+}$macrophages were present in both $\mathrm{CRC}$ tumours (Figure 3). Thus, we speculated the patient would benefit from ICIs based on her TIME characteristics. Notably, some metastatic CRC (mCRC) patients with MSI-H have primary resistance to ICIs. According to data from Keynote-177, the progression-free survival (PFS) curves for ICI therapy and chemotherapy crossed before 6 months. Considering that immunotherapy might take effect slowly, the proportion of patients with disease 

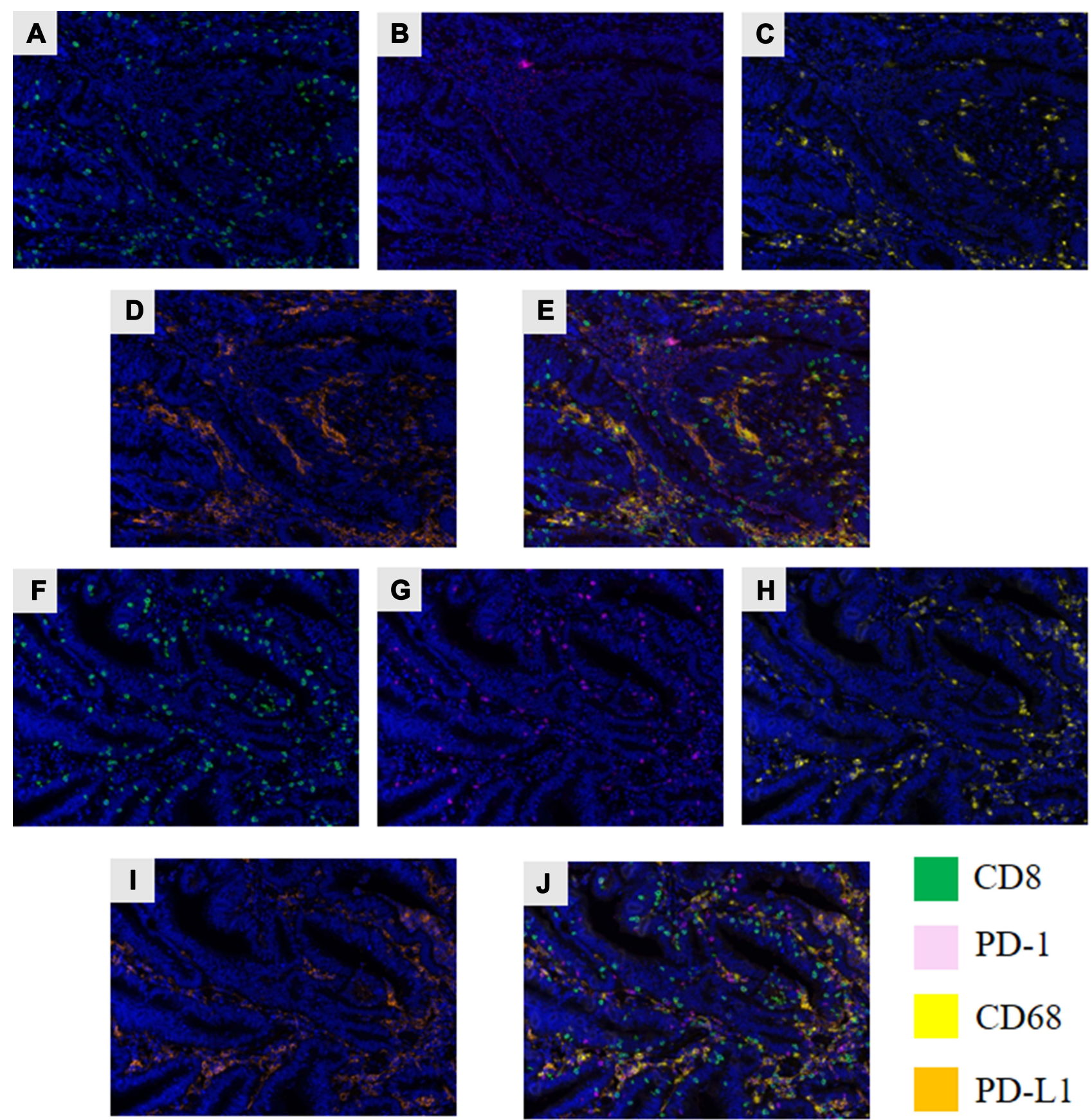

CD68

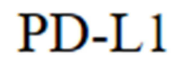

Figure 3 Assessment of the TIME via multiplex IHC (mlHC). (A and $\mathbf{F}$ ) The green fluorescence indicates CD8 ${ }^{+}$T cells. (B and $\left.\mathbf{G}\right)$ The pink fluorescence indicates PD-I expression. ( $\mathbf{C}$ and $\mathbf{H}$ ) The yellow fluorescence indicates CD68 expression. (D and $\mathbf{I}$ ) The Orange fluorescence indicates PD-LI expression. (E and $\mathbf{J})$ The pictures show all layers merged.

progression in the whole first-line treatment group was significantly higher than that in standard chemotherapy/ targeted therapy group $(29.4 \%$ vs $12.3 \%)$, indicating that some patients were resistant to ICIs. It is necessary to distinguish the MSI-H population in this group. ${ }^{18}$ The expression of PD-L1 has shown limited utility in predicting the effect of ICIs. However, TMB might be a more significant predictor. It is generally believed that tumours with higher mutation load can produce more neoantigens and thus have more immunogenicity and better response to immunotherapy. ${ }^{19}$ In our case, both CRC tumours had similar high TMB levels, suggesting potential efficacy of immunotherapy. However, when tumour relapse or metastasis occurs, determination of the feasibility of ICI strategies should be based on re-biopsy in order to confirm the MSS/MSI status. Combination therapies (anti-vascular 
therapies + ICIs) may be an effective treatment for these patients.

Awareness of such information will be helpful in the assessment of patients with CRC at risk of having divergent MMR/MSI status and TIME type. In addition, how to increase or reinforce the efficacy of immunotherapy, the optimal duration and combinations of treatments and the indications for surgical interventions after immunotherapy will be determined with time. ${ }^{20}$ Here, Assessing the MSS/ MSI status and TIME type in preoperative endoscopic biopsy specimens can be used to optimize the preoperative ICI strategy to reduce the scope of surgery and preserve organs. Additionally, clarifying genealogical information and family history is beneficial for the diagnosis of patients and the early prevention and treatment of unaffected family members. In conclusion, the effects of different MMR/MSI statuses and TIME types in CRC tumours on immunotherapy efficacy remain to be determined in further investigations.

\section{Abbreviations}

NGS, next-generation sequencing; MSI, microsatellite instability; LS, Lynch syndrome; CRC, colorectal cancer; TMB, tumour mutation burden; MSS, microsatellite stable; ICIs, immune checkpoint inhibitors.

\section{Data Sharing Statement}

The data used to support the findings of this study are available from the corresponding author upon request.

\section{Informed Consent}

The institutional review board of West China hospital, Sichuan University approved the use of health data in this research, and approved the publication of this case report. And informed consent was also obtained from the patient and the family for the publication of this case report.

\section{Acknowledgment}

We would like to acknowledge the Genecast Biotechnology Co., Ltd. for their assistance with the DNA NGS sequencing work, including sample preparation, library construction, exome capture, next-generation sequencing and bioinformatics analysis.

\section{Author Contributions}

Conception and study design:Qiheng Gou, Yali Shen. Execution:Qiheng Gou, Yuxin Xie. Acquisition of data:
Qiheng Gou, Yuxin Xie, Mengni Zhang, Ye Chen. Analysis and interpretation: Qiheng Gou. Writingoriginal draft: Qiheng Gou. Revision:Qiheng Gou, Yuxin Xie, Mengni Zhang, Ye Chen,Yali Shen. All authors reviewed, agreed on all versions of the article, agreed to take responsibility and be accountable for the contents of the article, and have agreed on the journal to which the article will be submitted.

\section{Funding}

This work was supported by the National Natural Science Foundation of China (81902723) and Sichuan Science and Technology Support Project (2019YJ0070).

\section{Disclosure}

The authors have no conflicts of interest to declare.

\section{References}

1. Chen W, Zheng R, Baade PD, et al. Cancer statistics in China, 2015. CA Cancer J Clin. 2016;66(2):115-132. doi:10.3322/caac.21338

2. Lynch HT, Snyder CL, Shaw TG, Heinen CD, Hitchins MP. Milestones of lynch syndrome: 1895-2015. Nat Rev Cancer. 2015;15(3):181-194. doi:10.1038/nrc3878

3. Hampel H, Frankel WL, Martin E, et al. Screening for the Lynch syndrome (hereditary nonpolyposis colorectal cancer). $N$ Engl J Med. 2005;352(18):1851-1860. doi:10.1056/NEJMoa043146

4. Ribic CM, Sargent DJ, Moore MJ, et al. Tumor microsatellite-instability status as a predictor of benefit from fluorouracil-based adjuvant chemotherapy for colon cancer. $N$ Engl J Med. 2003;349(3):247-257. doi:10.1056/NEJMoa022289

5. Sargent DJ, Marsoni S, Monges G, et al. Defective mismatch repair as a predictive marker for lack of efficacy of fluorouracil-based adjuvant therapy in colon cancer. $J$ Clin Oncol. 2010;28 (20):3219-3226. doi:10.1200/JCO.2009.27.1825

6. Le DT, Durham JN, Smith KN, et al. Mismatch repair deficiency predicts response of solid tumors to PD-1 blockade. Science. 2017;357(6349):409-413. doi:10.1126/science.an6733

7. Le DT, Uram JN, Wang H, et al. PD-1 blockade in tumors with mismatch-repair deficiency. $N$ Engl $J$ Med. 2015;372 (26):2509-2520. doi:10.1056/NEJMoa1500596

8. Wood LD, Parsons DW, Jones S, et al. The genomic landscapes of human breast and colorectal cancers. Science. 2007;318 (5853):1108-1113. doi:10.1126/science. 1145720

9. Carneiro da Silva F, Ferreira JRDO, Torrezan GT, et al. Clinical and molecular characterization of brazilian patients suspected to have lynch syndrome. PLoS One. 2015;10(10):e0139753. doi:10.1371/ journal.pone. 0139753

10. Sjursen W, Haukanes BI, Grindedal EM, et al. Current clinical criteria for Lynch syndrome are not sensitive enough to identify MSH6 mutation carriers. J Med Genet. 2010;47(9):579-585. doi:10.1136/jmg.2010.077677

11. Zhang X, Li J. Era of universal testing of microsatellite instability in colorectal cancer. World J Gastrointest Oncol. 2013;5(2):12-19. doi:10.4251/wjgo.v5.i2.12

12. Stjepanovic N, Moreira L, Carneiro F, et al. Hereditary gastrointestinal cancers: ESMO Clinical Practice Guidelines for diagnosis, treatment and follow-up ${ }^{\dagger}$. Ann Oncol. 2019;30(10):1558-1571. doi:10.1093/annonc/mdz233 
13. Richards S, Aziz N, Bale S, et al. Standards and guidelines for the interpretation of sequence variants: a joint consensus recommendation of the American college of medical genetics and genomics and the association for molecular pathology. Genet Med. 2015;17 (5):405-424. doi:10.1038/gim.2015.30

14. Overman MJ, McDermott R, Leach JL, et al. Nivolumab in patients with metastatic DNA mismatch repair-deficient or microsatellite instability-high colorectal cancer (CheckMate 142): an open-label, multicentre, Phase 2 study. Lancet Oncol. 2017;18(9):1182-1191. doi:10.1016/S1470-2045(17)30422-9

15. Salgado R, Denkert C, Demaria S, et al. The evaluation of tumor-infiltrating lymphocytes (TILs) in breast cancer: recommendations by an International TILs Working Group 2014. Ann Oncol. 2015;26(2):259-271. doi:10.1093/annonc/mdu450

16. Mehnert JM, Monjazeb AM, Beerthuijzen JMT, Collyar D, Rubinstein L, Harris LN. The challenge for development of valuable immuno-oncology biomarkers. Clin Cancer Res. 2017;23 (17):4970-4979. doi:10.1158/1078-0432.CCR-16-3063
17. Catacchio I, Scattone A, Silvestris N, Mangia A. Immune prophets of lung cancer: the prognostic and predictive landscape of cellular and molecular immune markers. Transl Oncol. 2018;11(3):825-835. doi:10.1016/j.tranon.2018.04.006

18. André T, Shiu KK, Kim TW, et al. Pembrolizumab in microsatellite-instability-high advanced colorectal cancer. $N$ Engl J Med. 2020;383(23):2207-2218. doi:10.1056/NEJMoa2017699

19. Schrock AB, Ouyang C, Sandhu J, et al. Tumor mutational burden is predictive of response to immune checkpoint inhibitors in MSI-high metastatic colorectal cancer. Ann Oncol. 2019;30(7):1096-1103. doi:10.1093/annonc/mdz134

20. Emens LA, Ascierto PA, Darcy PK, et al. Cancer immunotherapy: opportunities and challenges in the rapidly evolving clinical landscape. Eur J Cancer. 2017;81:116-129. doi:10.1016/j. ejca.2017.01.035

\section{Publish your work in this journal}

Cancer Management and Research is an international, peer-reviewed open access journal focusing on cancer research and the optimal use of preventative and integrated treatment interventions to achieve improved outcomes, enhanced survival and quality of life for the cancer patient.
The manuscript management system is completely online and includes a very quick and fair peer-review system, which is all easy to use. Visit http://www.dovepress.com/testimonials.php to read real quotes from published authors. 